\title{
Antimicrobial-resistance genes in the human gut prior the antibiotic- therapy era
}

Created by: Tasha Santiago-rodriguez

Version received: 3 June 2019

Antibiotic-resistance has long been associated with the use and abuse of antibiotics. However, increasing evidence is suggesting that antibiotic-resistance is in fact a phenomenon that has been occurring in natural environments for thousands and possibly millions of years. With the expansion of the microbiome field, it is now possible to characterize antibiotic-resistance genes altogether in different samples, including the human gut. This has also enabled the characterization of ancient human gut microbiomes, which also include antibiotic-resistance genes. Mummified gut remains represent a unique opportunity to characterize the microbiome and antibiotic-resistance genes prior the antibiotic-therapy era. Surprisingly, mummies from the Inca and Italian nobility cultures showed to possess antibioticresistance-like genes similar to modern-day antibiotic-resistance genes conferring resistance to beta-lactams, sulfa, quinolones and vancomycin, just to mention a few examples. This is intriguing as it further supports that antibioticresistance began in the environment and was transferred to the human gut by means that remain to be investigated and are a matter of ongoing speculation.

\section{What is the process of mummification?}

Mummification is the process of preserving a body by either artificial or natural methods.Artificial mummification, also known as embalming, is when a body is intentionally preserved by removing visceras and filled with substances that would aid in its preservation. The process of artificial mummification in ancient Egypt is perhaps one of the most wellknown examples. The process of natural mummification, on the other hand, is when a body is preserved by being exposed to a combination of environmental conditions including low oxygen levels, dry and cold weather, and low or high temperatures. Visceras are not necessarily removed and the body is not filled with substances that would aid in the preservation of the body, as in the case of artificial mummification [1].

Ancient cultures in South America, including thelncas, and nobility in Italy practiced the process of natural mummification. Inca bodies were wrapped using several layers of textiles and were bound with cords (Figure 1). The body would then be placed in spaces or chambers where environmental conditions were optimal for the process of natural mummification to occur. These spaces or chambers would be often reopened to add more bodies as needed. Other bodies, particularly victims of sacrifices, would be placed outside, where environmental conditions were also optimal for the process of natural mummification to occur (Figure 1) [2]

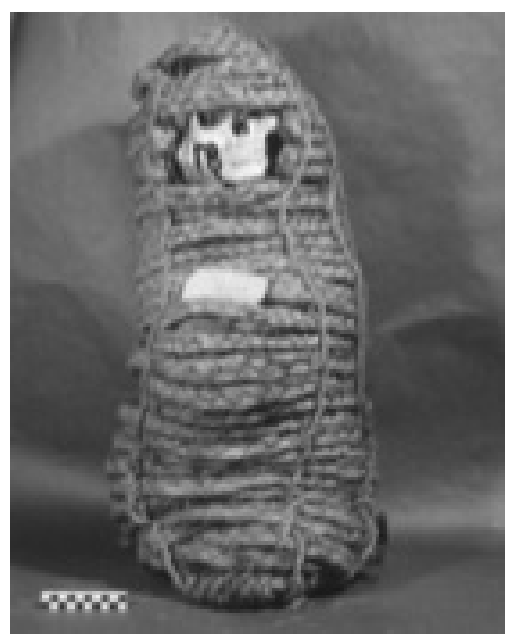

Figure 1. Inca mummy in fetal position and wrapped with cords. While her face was skeletonized, her body was in a good state of preservation. 


\section{Encyclopedia}

The process of natural mummification in Italy differed from that practiced by the Incas in that fluids would be removed before placing the body in rooms or chambers. This process of "dripping" the body was essential to stop or minimize the process of putrefaction aid by microorganisms. Natural mummification of Italian nobility bodies may have been favored by the micro-climatic conditions of certain regions in Italy [1].

\section{What is the human microbiome and why study it in mummies?}

Just until recently, the concept of the humanmicrobiome was applied to understand ancient cultures. The human microbiome refers to the commensal, symbiotic and pathogenic bacteria, viruses, archaea, and lower eukaryotes and all of the genes necessary for their function and survival, by dynamically interacting with each other and the host [3]. Since the body is preserved during the process of natural mummification and environmental conditions halt the process of putrefaction, it is feasible to hypothesize that known members of the gut microbiome would also be preserved. It is also feasible to hypothesize that the microbiome of mummies would be a hybrid between the gut microbiome as known in today, as well as the thanatomicrobiome, which refers to the microbiome during the process of decomposition. In addition, since the gut microbiome usually reflects dietary habits, lifestyles, comorbidities and pathologies [4], direct characterization of mummified gut remains represents a unique opportunity to study of the evolution of the gut microbiome in association with the mentioned factors. Characterization of ancient gut microbiomes would also further expand the understanding of ancient South American and European cultures. A similar approach can be used to understand the gut microbiome in a number of other extinct and ancient cultures.

Mummies that had their gut microbiomes characterized are unique in that they have shown various pathologies including Chagas disease and leishmaniasis, as in the case of Inca mummies recovered from the region of what is known as Cuzco, the ancient capital of Peru. Other pathologies included colorectal cancer, bronchopneumonia and cirrosis, as in the case of the Italian nobility mummies, which were recovered in Naples, Italy. The characterized mummies also had different lifestyles, dietary habits, and socioeconomic status. For instance, the Incas had a stratified society, where the farmers were the most common and most numerous, followed by the nobility and the Emperor. Most Inca people were in a constant and direct contact with the environment because they were accomplished travelers, builders and farmers. Italian nobility, on the other hand, had minimal contact with the environment [5]

Characterization of the gut microbiomes of Inca and Italian nobility mummies also enabled the opportunity to understand its association with ancient diets. For instance, the Inca diet included quinoa, maize, and potatoes. While meat was rarely consumed, it may have included guinea pigs, llama and alpaca herds, which were consumed as jerky. Fermented maize, quinoa or other grains were consumed as drinks, also known as chicha. Diets of Italian nobility, on the other hand, may have been primarily animal-based. This is known based on historical and isotopic records that have shown that meat was essential in the Italian nobility diets. Other food items included fish, which was consumed on penitential occasions, eggs and cheese. Unlike the Incas, fruits and vegetables were not commonly included in the Italian nobility's diet $[6]$.

The seemingly contrasting dietary habits and lifestyles of the Incas and Italian nobility opened the opportunity to understand the gut microbiome and how it could reflect these differences in ancient cultures.

\section{How does studying the gut microbiome leads to study antibiotic-resistance genes in mummies?}

One approach to characterize the microbiome is to determine the DNA sequences of all the microorganisms in a sample. All the DNA sequences includes, not only those that facilitate the identification of the microbes that existed in the mummies guts, but also those genes essential for function and survival, including antibiotic-resistance. Antibioticresistance is often associated with the extensive use of antibiotics in medicine and livestock, which has probably resulted in a faster evolution of antibiotic-resistant bacteria in various environments, including the human gut. The characterization of the gut microbiome of Inca and Italian nobility mummies, with contrasting differences in diets and lifestyles, also opens the opportunity to understand the evolution of antibiotic-resistance. Why? Antibiotic-resistance, in fact, is not a new phenomenon. Evidence has found beta-lactamases, which confer resistance to beta-lactams, in thousands-year old permafrost [7], indicating that antibiotic-resistance is not a recent event. Other studies have found antibiotic-resistance genes in isolated regions with no known anthropological impact [8]. 
Antibiotic-resistance-like genes have also been identified in the gut of Inca and Italian nobility mummies Figure 2).

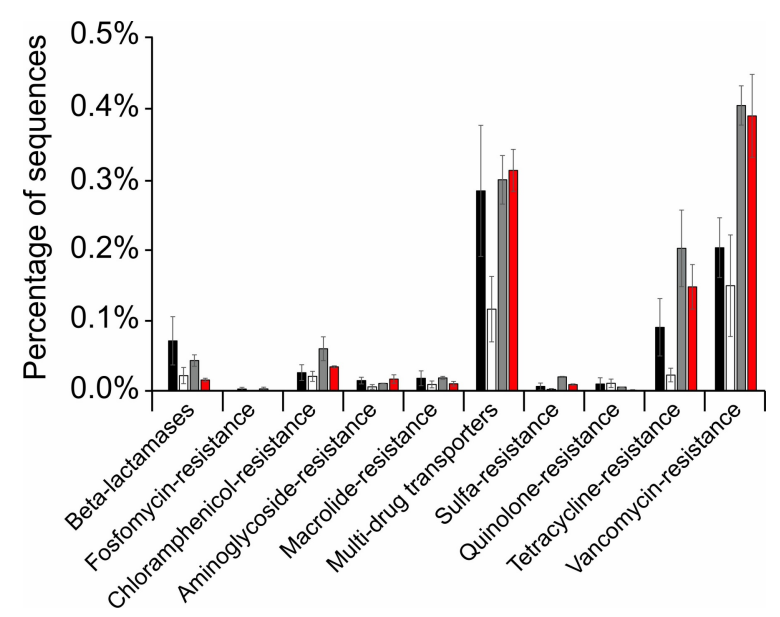

Figure 2. Bar plot of several of the antibiotic-resistance genes identified in the Inca mummies (black bars) and Italian nobility mummies (white bars). Results were also compared to modern Amazonians (gray bars) and modern Italians (red bars).

How did antibiotic-resistance reached the mummies guts? One hypothesis is that antibiotic-resistance genes reached the human gut by direct consumption of soil-contaminated crops (Figure 3). Soil microbes, including Streptomyces spp. for example, are known to produce a number of antimicrobials, including streptomycin, possibly as a metabolic product or to compete for resources including nutrients, space and oxygen. Thus, it is reasonable to hypothesize that neighboring bacteria may have developed resistance to antibiotic and antibiotic-like molecules produced by microbes,

like Streptomyces spp.

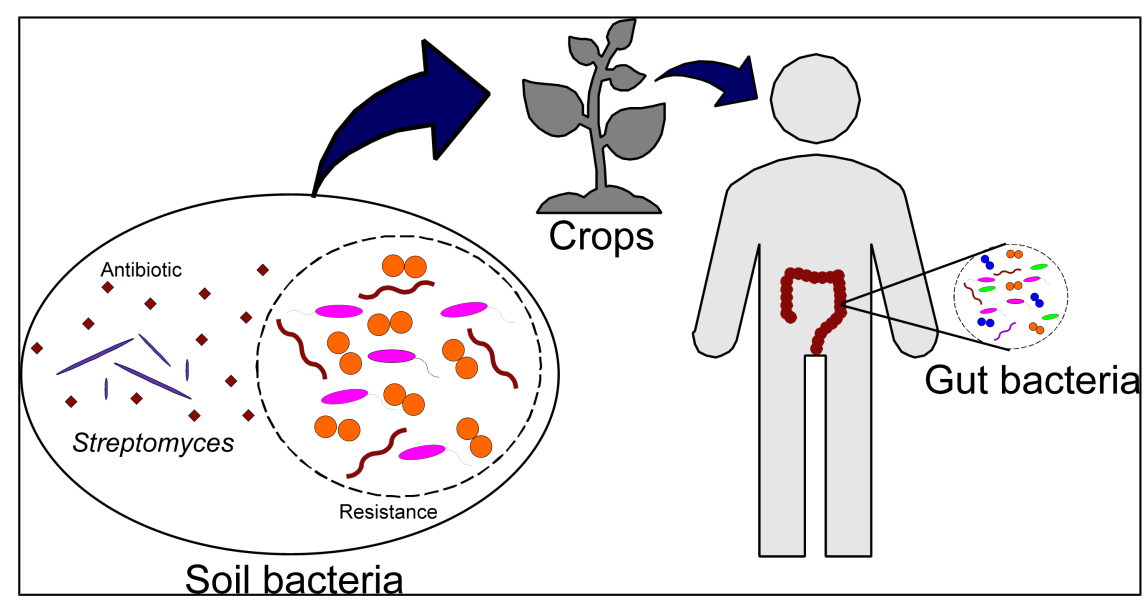

Figure 3. Hypothesis of how antibiotic-resistance genes reached the human gut.

\section{Future directions}

As we continue to understand the microbiomes of ancient cultures, along with the genes necessary for their survival, including antibiotic-resistance genes, we will have a better understanding of our microbiomes, how they have evolved in relation to our lifestyles, diets, disease and disease treatment, including antibiotic treatment. By looking at the microbiomes and antibiotic-resistance genes of ancient cultures we may better predict microbiome and antibioticresistance evolution.

\section{References}

1. Andrew T. Chamberlain; The scientific study of mummies. International Journal of Osteoarchaeology 2003, 13, 393-394, 10.1002/oa.681.

2. Vreeland, J.M. Mummies of Peru; Cockburn, A., Cockburn, E., and Reyman, T.A., Eds.; Cambridge University Press: United Kingdom, 1998; pp. 154-189. 
3. Ilseung Cho; Martin J. Blaser; The human microbiome: at the interface of health and disease. Nature Reviews Genetics 2012, 13, 260$270,10.1038 / \mathrm{nrg} 3182$.

4. Lawrence A David; Arne C Materna; Jonathan Friedman; Maria I Campos-Baptista; Matthew C Blackburn; Allison Perrotta; Susan E Erdman; Eric J Alm; Host lifestyle affects human microbiota on daily timescales. Genome Biology 2014, 15, R89, 10.1186/gb-2014-15-7r89.

5. Tasha M. Santiago-Rodriguez; Gino Fornaciari; Stefania Luciani; Gary A. Toranzos; Isolina Marota; Valentina Giuffra; Raul J. Cano; Gary Toranzos; Gary A Toranzos; Gut Microbiome and Putative Resistome of Inca and Italian Nobility Mummies.. Genes 2017, 8, 310, 10.3390/genes8110310.

6. Gino Fornaciari; Food and disease at the Renaissance courts of Naples and Florence: A paleonutritional study. Appetite 2008, 51, 1014, 10.1016/j.appet.2008.02.010.

7. Vanessa M. D'Costa; Christine E. King; Lindsay Kalan; Mariya Morar; Wilson W. L. Sung; Carsten Schwarz; Duane Froese; Grant Zazula; Fabrice Calmels; Regis Debruyne; et al.G. Brian GoldingHendrik N. PoinarGerard D. Wright Antibiotic resistance is ancient. Nature 2011, 477, 457-461, 10.1038/nature10388.

8. Kirandeep Bhullar; Nicholas Waglechner; Andrew Pawlowski; Kalinka Koteva; Eric D. Banks; Michael D. Johnston; Hazel A. Barton; Gerard D. Wright; Antibiotic Resistance Is Prevalent in an Isolated Cave Microbiome. PLoS ONE 2012, 7, e34953, 10.1371/journal.pone.0034953.

\section{Keywords}

antibiotic-resistance genes; Incas; Italian nobility; microbiome; mummies

(C) 2019 by the author(s). Distribute under aCreative Commans CC BY license 